\title{
From exome analysis in idiopathic azoospermia to the identification of a high-risk subgroup for occult Fanconi anemia
}

\author{
Csilla Krausz, MD, PhD 1,2, Antoni Riera-Escamilla, $\mathrm{PhD}^{2}$, Chiara Chianese, $\mathrm{PhD}^{2}$, \\ Daniel Moreno-Mendoza, $\mathrm{MD}^{2}$, Elisabet Ars, $\mathrm{PhD}^{2}$, Osvaldo Rajmil, MD, $\mathrm{PhD}^{2}$, Roser Pujol, $\mathrm{PhD}^{3}$, \\ Massimo Bogliolo, $\mathrm{PhD}^{3}$, Ignacio Blanco, $\mathrm{MD}, \mathrm{PhD}^{4}$, Ines Rodríguez, $\mathrm{MD}^{4}$, Isabel Badell, $\mathrm{MD}, \mathrm{PhD}^{5}$, \\ Eduard Ruiz-Castañé, $\mathrm{MD}^{2}$ and Jordi Surrallés, $\mathrm{PhD}^{3}$
}

Purpose: In about $10 \%$ of patients affected by Fanconi anemia (FA) the diagnosis is delayed until adulthood, and the presenting symptom in these "occult" FA cases is often a solid cancer and cancer treatment-related toxicity. Highly predictive clinical parameter(s) for diagnosing such an adult-onset cases are missing.

Methods: (1) Exome sequencing (ES), (2) Sanger sequencing of FANCA, (3) diepoxybutane (DEB)-induced chromosome breakage test.

Results: ES identified a pathogenic homozygous FANCA variant in a patient affected by Sertoli cell-only syndrome (SCOS) and in his azoospermic brother. Although they had no overt anemia, chromosomal breakage test revealed a reverse somatic mosaicism in the former and a typical FA picture in the latter. In 27 selected SCOS cases, 1 additional patient showing compound heterozygous pathogenic FANCA variants was identified with positive chromosomal breakage test.
Conclusion: We report an extraordinarily high frequency of FA in a specific subgroup of azoospermic patients (7.1\%). The screening for FANCA pathogenic variants in such patients has the potential to identify undiagnosed FA before the appearance of other severe clinical manifestations of the disease. The definition of this highrisk group for "occult" FA, based on specific testis phenotype with mild/borderline hematological alterations, is of unforeseen clinical relevance.

Genetics in Medicine (2019) 21:189-194; https://doi.org/10.1038/ s41436-018-0037-1

Keywords: Exome sequencing; Genetics; Male infertility; Fanconi anemia; Azoospermia

\section{INTRODUCTION}

The severest form of male factor infertility is nonobstructive azoospermia (NOA), which occurs in approximately $1 \%$ of all men of reproductive age and in the majority of patients the etiology remains unknown. ${ }^{1}$ Because spermatogenesis is regulated by the concerted action of $>2000$ genes, a large proportion of idiopathic NOA might be attributable to a not yet identified genetic defect. ${ }^{2}$ Within the past few years, exome sequencing (ES) has proved to be a powerful tool for the discovery of causative variants in familial cases of NOA. ${ }^{3-10}$ On the other hand, this approach has the potential to incidentally reveal undiagnosed diseases. Genes involved in stem cell proliferation and DNA repair are important not only for spermatogenesis but also for other physiological processes. For instance, the association between Fanconi anemia (FA, OMIM 227650), caused by pathogenic variants in genes involved in DNA interstrand cross-link repair, and infertility is well known in humans; however, the exact testicular phenotype in FA patients has not been explored. FA arises in the large majority of cases with bone marrow failure during childhood, however, in $10 \%$ of cases the diagnosis is delayed until adulthood. ${ }^{11}$ The late diagnosis frequently occurs in those patients who present a slow progressive depletion of bone marrow hence the sign of onset may be the appearance of a cancer instead of an overt anemia. Diagnosing occult FA has a relevance also concerning cancer treatment due to acute toxicity to chemotherapy. We performed ES in an idiopathic NOA (iNOA) patient with consanguineous parents, who displayed Sertoli cell-only syndrome (SCOS). Our investigation revealed a pathogenic variant in the FANCA gene providing an incidental diagnosis of FA during a genetic investigation for NOA in an adult man. Based on this finding we revisited all our patients with similar testis histology and selected

\footnotetext{
${ }^{1}$ Department of Experimental and Clinical Biomedical Sciences "Mario Serio", Centre of Excellence DeNothe, University of Florence, Florence, Italy; ${ }^{2}$ Andrology Department, Fundació Puigvert, Universitat Autònoma de Barcelona, Instituto de Investigaciones Biomédicas Sant Pau (IIB-Sant Pau), Barcelona, Catalonia, Spain; ${ }^{3}$ Genetics Department and Biomedical Research Institute, Hospital de Sant Pau, Center for Biomedical Research on Rare Diseases (CIBERER), and Department of Genetics and Microbiology, Universitat Autònoma de Barcelona, Barcelona, Catalonia, Spain; ${ }^{4}$ Hospital Germans Trias i Pujol, Badalona, Catalonia, Spain; ${ }^{5}$ Pediatrics Department, Hospital de Sant Pau, Barcelona, Catalonia, Spain. Correspondence: Csilla Krausz (csilla.krausz@unifi.it) or Jordi Surrallés (jsurralles@santpau.cat)

These authors contributed equally: Csilla Krausz, Antoni Riera-Escamilla.
} 
among them those with mild alterations or borderline values of platelets and mean corpuscular volume (MCV) for further screening of FANCA gene pathogenic variants. This approach has led to the identification of a high-risk group for adultonset FA.

\section{Study populations}

\section{MATERIALS AND METHODS}

Study population I: A 43-year-old man (04-170) with iNOA and with consanguineous parents (first-cousin marriage) was selected for ES. All known causes of azoospermia were excluded. Testis histology revealed SCOS type I (no germ cells were present in the tubules). See Table 1.

Study population II: 27 iNOA patients with testis histology of SCOS type I and SCOS type II (SCOS with germ cells in a minority of tubules), with platelets $<200,000 / \mathrm{L}$ and MCV $>85$ $\mathrm{fL}$ were selected for FANCA pathogenic variant screening (Table S1). The local ethical committee of the Fundació Puigvert approved the study (2014/04c) and all patients signed the informed consents approved by the ethical committee.

\section{Exome sequencing in patient 04-170}

Library preparation, Bioinformatic analysis and variants filtering are described in Supplementary Material.

\section{Multiplex ligation-dependent probe amplification}

Large deletions were analyzed using a multiplex ligationdependent probe amplification (MLPA) kit developed by MRC-Holland BV as we described before. ${ }^{12}$

\section{FANCA Sanger sequencing analysis}

Two-step Sanger sequencing (details in Castella et al. 2011) ${ }^{12}$ in 27 selected iNOA patients: first, the three most commonly mutated exons (exons 13,36,38) in the Spanish population; second, the remaining FANCA exons.

\section{Diepoxibutane (DEB)-induced chromosomal breakage test} Pathogenic variant carrier NOA patients and and their brother's peripheral blood lymphocytes, as described elsewhere $^{13}$ (see also Supplementary Material).

\section{Gene expression evaluation}

Quantitative reverse transcription polymerase chain reaction (qRT-PCR) analysis was performed to evaluate FANCA expression in adult testes biopsies, collected in our clinic and characterized with molecular markers for the definition of distinct germ cell types (details in Supplementary Material and Table S2).

\section{RESULTS}

\section{Exome analysis of a NOA patient affected by SCOS}

Patient 04-170 carried a total of 20,477 exonic variants. Following standard filtering (described in Supplementary Material) and the exclusion of synonymous variants with a minor allele frequency $(\mathrm{MAF}) \geq 0.05$, we filtered for all homo/ hemizygous variants that presented an in-house index of pathogenicity (IP) $\geq 0.7$. Of the eight resulting variants, only one mapped to a gene with proved implication in spermatogenesis (FANCA) and presented the highest pathogenic score $(\mathrm{IP}=0.9$,) $\quad$ (Fig. 1, Figure S1 and Table S3). The c. $2639 \mathrm{G}>\mathrm{A}$ variant maps to exon 28 of the FANCA gene (NM_000135.2) and its pathogenicity was proven by functional studies. ${ }^{12,14}$ It was already shown to cause FA in a Spanish patient, ${ }^{12}$ as well as reported in the FA Mutation Database at Rockefeller University both in homozygosis and heterozygosis in eight FA patients (Table S4). DEB-induced chromosomal breakage test, one of the gold-standard tests used in the routine practice for the diagnosis of FA, gave a positive result in a fraction of cells, indicating FA somatic mosaicism (Fig. 1b). The presence of $2-3 \%$ of wild-type alleles in the next-generation sequencing (NGS) data (data not shown) suggests a possible mechanism of genetic reversion by back mutation. The confirmation of this hypothesis would request additional experiments using digital PCR, cloning, and single-clone sequencing or amplicon analysis by deep NGS. ${ }^{15}$

Following the discovery of the variant, the patient, during genetic counseling, informed the doctor that his brother also suffered from azoospermia. The screening of FANCA in his brother revealed the same genotype. The man could not undergo physical examination in our clinic due to geographic distance, but according to the family doctor he has no evident skeletal abnormalities or other FA-related symptoms. DEBinduced chromosomal breakage test showed that the majority of cells resulted positive, revealing a typical complete FA picture (Fig. 1b). It is interesting to note that although this man presented a mild decrease of platelets in 2011, and in 2013 also displayed a mild decrease of red blood cells and leukocytes, this condition was not further explored by the family doctor. The hemogram from 2016, requested after our diagnosis, shows a pronounced decrease of all three cell types (Table 1).

\section{FANCA pathogenic variant screening in selected iNOA patients with SCOS}

From our large cohort of 1300 infertile men, 136 were classified as iNOA with testis histology of SCOS type I or type II. For 120/ 136 patients hematological parameters were available and we selected those $(n=27)$ with platelets $<200,000 / \mathrm{L}$ and MCV $>85$ fL (Fig. S2, Table S1). Genomic DNA from peripheral blood of this specific group of 27 iNOA patients was screened for pathogenic variant firstly in exons 13,36 , and 38 , i.e., those exons that contain the most commonly observed pathogenic variants in Spanish FA patients and for large FANCA deletions through MLPA. We identified a heterozygous variant in exon 38 in patient 14-339 [NM_000135.2: c.3788_3790delTCT, p.Phe1263del)]. This analysis was followed by Sanger sequencing of the entire FANCA gene, which revealed a second heterozygous variant in exon 39 [NM_000135.2:c.3913C>T,p.Leu1305Phe] in patient 14-339. Both pathogenic variants were previously reported in many 


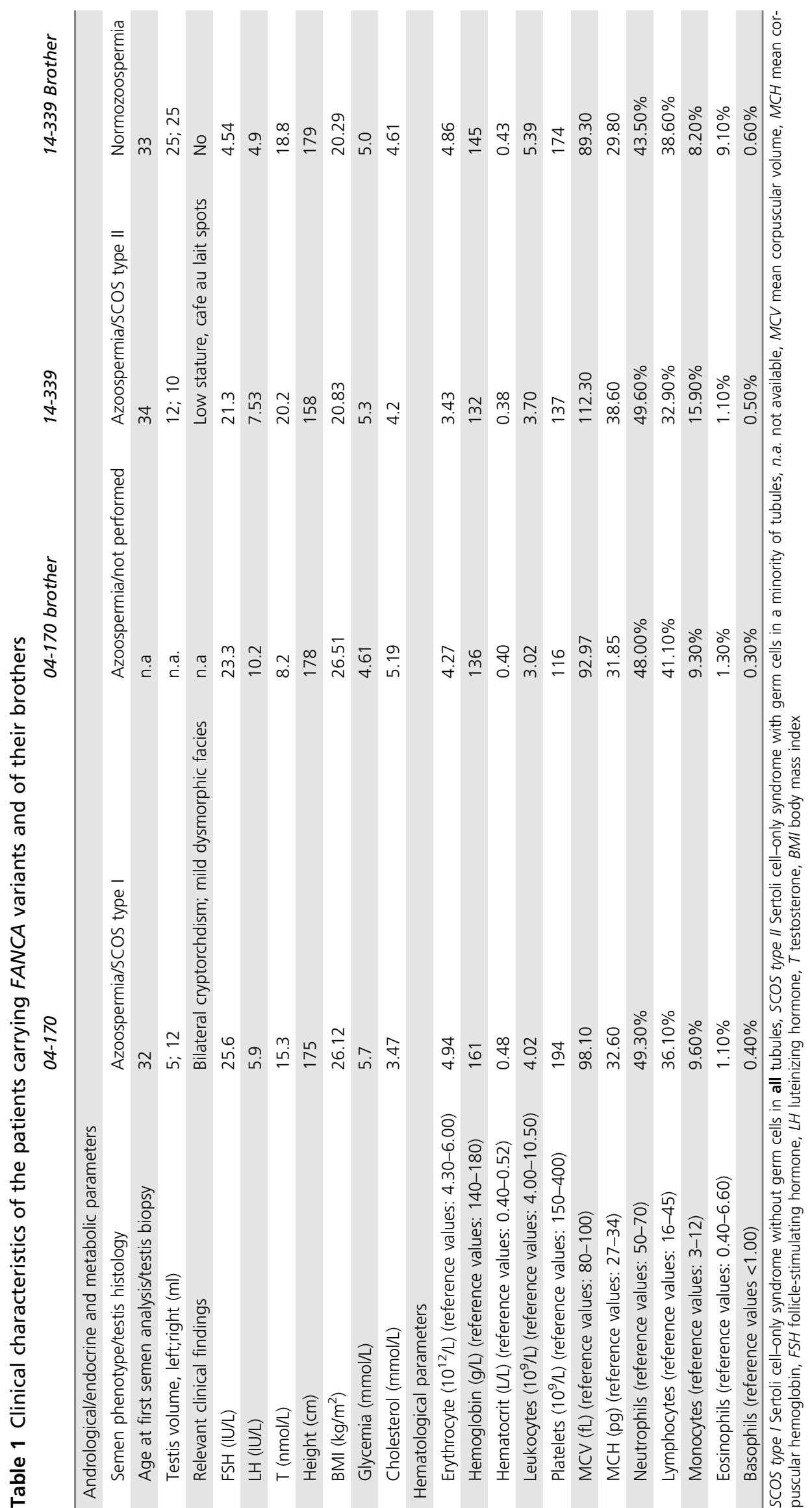




\section{a}

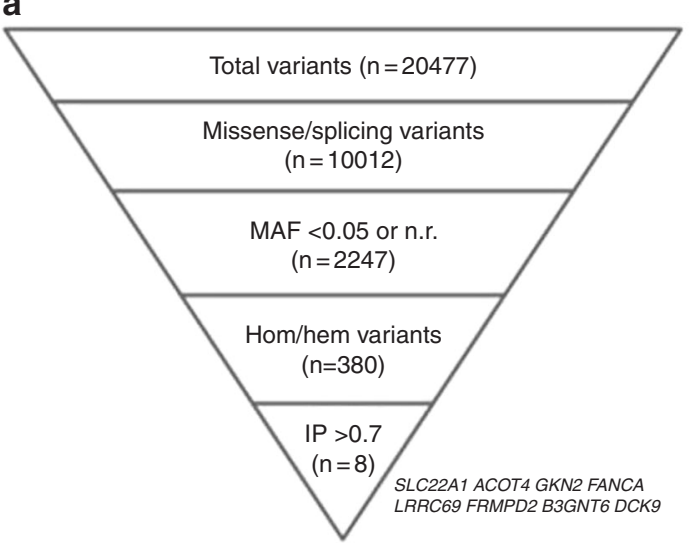

b

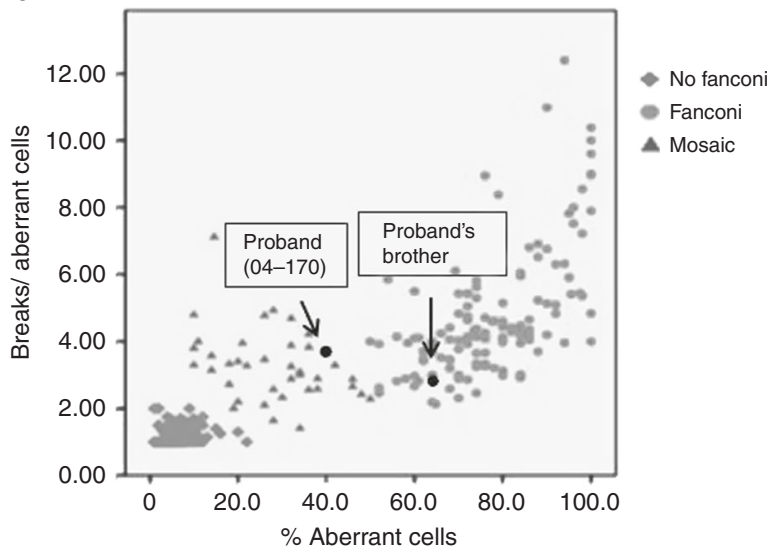

C

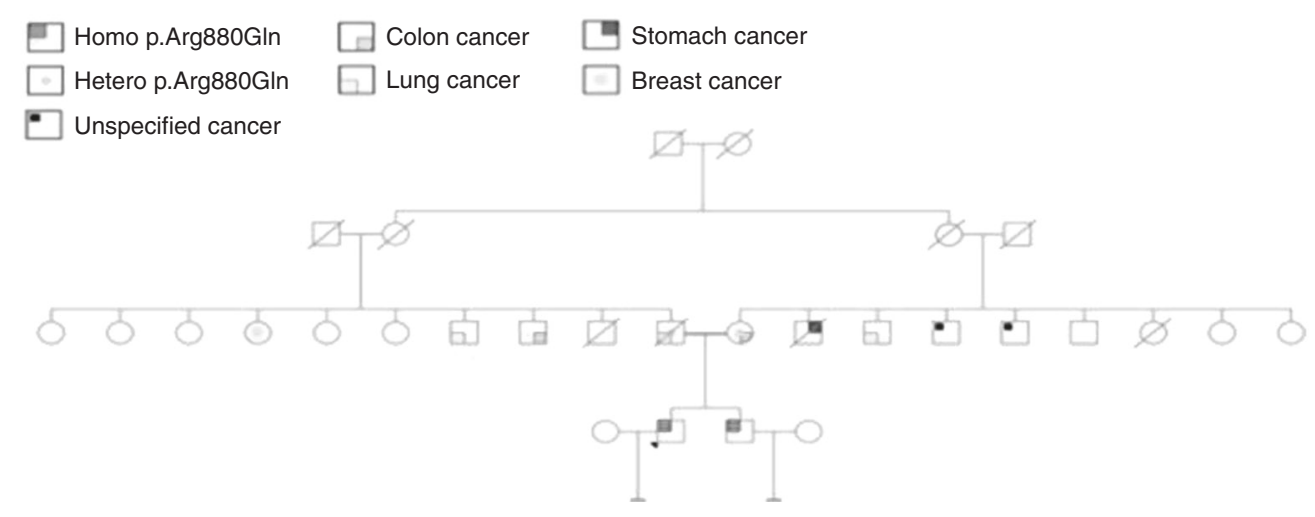

Fig. 1 Investigation on the patient 04-170 and his brother, both carriers of the FANCA variant. a The inverted pyramid scheme indicates how ES data were filtered and prioritized for patient 04-170 starting from a total of 20,477 variants. Eight homozygous, rare, and potentially pathogenic variants were identified in eight different genes (in bold gene expressed in testis). b Graphic representation of the chromosomal breakage test for patient 04-170 and his brother. The arrows illustrate the patient's and his brother's position in relation to the distribution of historical data collected in our laboratory according to the percentage of DEB-induced aberrant cells and the number of breakages in each DEB-induced aberrant cell. In the No FA group, only individuals with at least one aberrant cell were included. c The pedigree structure shows the segregation of the $c .2639 C>T$ ( $p . A r g 880 G \ln )$ variant. The lower-left arrow indicates the proband 04-170 who was subjected to ES analysis whereas his brother's DNA was sequenced by Sanger sequencing. Our patient and his brother both carried the c.2639G>A (p.Arg880GIn) variant in homozygosis and were azoospermic. The parents' DNA samples were not available for testing, but because the variant is rare it can be assumed that the parents were both heterozygous carriers. MAF minor allele frequency, IP index of pathogenicity

FA patients and are clearly pathogenic (Fig. S2, Table S4). Similar to patient 04-170, DEB-induced chromosomal breakage test gave a positive result in a fraction of cells confirming mosaic FA (Fig. S3). The brother of this patient showed normozoospermia and his hormone levels, testis volume, and blood count were within the normal range (Table 1). Sanger sequencing revealed only one of the two variants in heterozygosis [NM_000135.2:c.3913C > T, p.Leu1305Phe].

\section{FANCA expression in the testis}

qRT-PCR analysis was performed in a collection of testis biopsy samples (not belonging to the patients' testis biopsies due to the lack of material recollection) previously characterized by a molecular genetic approach (see Supplementary Material). FANCA was undetectable in SCOS, but showed the highest expression in biopsies with a prevalent spermatogonial arrest (SGA) and obstructive azoospermia (i.e., presence of all spermatogenesis stages). This expression pattern is compatible with a prevalent expression in spermatogonial and early spermatocytic stage (Fig. S4).

\section{DISCUSSION}

The large majority of FA patients are diagnosed in childhood due to the presence of significant birth defects. In about $10 \%$ of patients the diagnosis is delayed until adulthood, ${ }^{11}$ especially when they appear normal or have subtle findings that may be overlooked. The late diagnosis frequently occurs in those patients who present a slow progressive depletion of bone marrow; hence the sign of onset may be the appearance of a cancer instead of an overt anemia. According to the FA Guidelines, ${ }^{16}$ "male infertility" is one of those adult-onset signs/symptoms that should raise suspicion of FA, hence should prompt clinicians to perform peripheral blood testing. However, while FA is a rare disease (prevalence of $1 / 160,000$ ), infertility is a common pathological condition, present in $7 \%$ 
of the general male population. Moreover, it is an extremely heterogeneous condition ranging from the complete absence of spermatozoa in the ejaculate (azoospermia) to decreased sperm number or motility/morphology. ${ }^{1}$ Even the NOA phenotype can be further subclassified according to the testis histology, e.g., complete absence of germ cells (SCOS), spermatogenic arrest (at different stages), or hypospermatogenesis. Therefore, referring to "infertility" as a possible FA sign is extremely broad and requires a more precise definition in the clinical practice.

Our first patient, diagnosed through ES, presented borderline values of blood counts and besides infertility showed another potential FA sign, mild dysmorphic facies. His brother with the same reproductive problem carried the same genotype and as expected, the chromosomal breakage test confirmed FA in both brothers i.e. a picture of mosaic FA in patient $04-170$ and a complete FA in his brother. The brother did not show dysmorphic features but displayed a progressive impairment of hematopoiesis reaching to pathologically low values at the control in 2016 (performed after our diagnosis).

The third patient presented low stature and some cafe au lait spots in his hands and back; however, FA was not suspected until he came to our clinic and was diagnosed with azoospermia. Similar to patient 04-170, DEB test also showed a picture of mosaic FA in this man. Patient 14-339's brother carried only one of the variants in heterozygosis and his testicular function was normal. A common feature of all three affected men were their testis phenotype, i.e., SCOS.

In this study, we report an unexpectedly high frequency of FA $(2 / 28=7.1 \%$ or $3 / 29=10.3 \%$ if we consider also the brother) in a specific subgroup of iNOA subjects with testicular phenotype of SCOS type I or type II and with slightly altered/borderline hematological parameters. Our study indicates that andrological evaluation, especially in SCOS patients, should not only include hormone measurement but also blood count, because it may lead to the diagnosis of unsuspected FA in adulthood. It further supports that FA is not exclusively a childhood illness, and diagnosis and treatment are no longer exclusively performed by pediatricians. We propose that the evaluation of hematological parameters, in particular platelet count and MCV in NOA patients, should be included in the routine andrological workup. Likewise, we provide a more precise andrological criteria to all clinicians, about the type of "infertility" subgroup in which the likelihood of FA is relatively high. The combined assessment of testis histology and hematological parameters allows the diagnosis of "occult" FA before the appearance of severe pathologies related to this disease (e.g., hematological malignancies and specific solid cancers). It has been reported that in more than $20 \%$ of patients with FA who developed solid tumors, the diagnosis of FA was made only after the appearance of their cancer and its treatment. ${ }^{17}$ (Some genotoxic anticancer agents are contraindicated in FA patients because of their DNA repair deficiency.) The diagnosis of FANCA variants in these three men also has relevance in case they develop this type of cancer in the future and thanks to our investigation, they are now receiving specific medical attention including strict follow-up by oncohematologists. The link between severe spermatogenic impairment and chronic diseases has been advocated by epidemiological observations, showing that infertile men (oligo/azoospermic) have a higher risk of morbidity (including cancer) and a lower life expectancy. ${ }^{18-20} \mathrm{~A}$ biological basis for such observations may be (1) the previously observed high CNV burden ${ }^{2}$ (indicating chromosomal instability) in infertile men, and (2) that some genes involved in spermatogenesis may also have a role in carcinogenesis (especially those involved in stem cell renewal/differentiation and mismatch repair mechanisms ${ }^{21}$ ). Besides its clinical importance, our study stimulates further investigations on the discovery of common genetic factors involved both in SCOS and bone marrow failure.

\section{ELECTRONIC SUPPLEMENTARY MATERIAL}

The online version of this article (https://doi.org/10.1038/s41436018-0037-1) contains supplementary material, which is available to authorized users.

\section{ACKNOWLEDGEMENTS}

This work was funded by the Spanish Ministry of Health Instituto Carlos III-FIS (grant number: FIS/FEDER-PI14/01250; PI17/01822) and European Commission, Reproductive Biology Early Research Training (REPROTRAIN, project number: 289880) awarded to CK and AR-E. Ente Cassa di Risparmio di Firenze. JS is funded by ICREA Academia, EUROFANCOLEN project HEALTH-F5-2012305421, CIBERER, and SAF2015-64152-R/FEDER. The authors wish to thank the patients participating in the study for their important collaboration. We also thank the clinicians from the Andrology Unit of the Fundació Puigvert, J. Sarquella and J. Sanchez-Curbelo. We acknowledge Esperança Martí, President of the Fundació Puigvert.

\section{DISCLOSURE}

The authors declare no conflicts of interest.

\section{REFERENCES}

1. Tournaye H, Krausz C, Oates RD. Novel concepts in the aetiology of male reproductive impairment. Lancet Diabetes Endocrinol. 2016:5:544-553.

2. Krausz C, Escamilla AR, Chianese $C$. Genetics of male infertility: from research to clinic. Reproduction. 2015;150:R159-74.

3. Ayhan Ö, Balkan M, Guven A, et al. Truncating mutations in TAF4B and ZMYND15 causing recessive azoospermia. J Med Genet. 2014;51: 239-44.

4. Okutman O, Muller J, Baert $Y$, et al. Exome sequencing reveals a nonsense mutation in TEX15 causing spermatogenic failure in a Turkish family. Hum Mol Genet. 2015;24:5581-8.

5. Ramasamy $R$, Bakırcıoğlu ME, Cengiz C, et al. Whole-exome sequencing identifies novel homozygous mutation in NPAS2 in family with nonobstructive azoospermia. Fertil Steril. 2015;104:286-91.

6. Gershoni M, Hauser R, Yogev L, et al. A familial study of azoospermic men identifies three novel causative mutations in three new human azoospermia genes. Genet Med. 2017;19:998-1006.

7. Kherraf Z-E, Christou-Kent M, Karaouzene T, et al. SPINK2 deficiency causes infertility by inducing sperm defects in heterozygotes and azoospermia in homozygotes. EMBO Mol Med. 2017;9:1132-49. 
8. Arafat M, Har-Vardi I, Harlev A, et al. Mutation in TDRD9 causes nonobstructive azoospermia in infertile men. J Med Genet. 2017;54:633-9.

9. Okutman O, Muller J, Skory V, et al. A no-stop mutation in MAGEB4 is a possible cause of rare $\mathrm{X}$-linked azoospermia and oligozoospermia in a consanguineous Turkish family. J Assist Reprod Genet. 2017;34:683-94.

10. Colombo R, Pontoglio A, Bini M. Two novel TEX15 mutations in a family with nonobstructive azoospermia. Gynecol Obstet Invest. 2017;82: 283-6.

11. Alter BP Bone marrow failure: a child is not just a small adult (but an adult can have a childhood disease). Hematol Am Soc Hematol Educ Progr. 2005;1:96-103.

12. Castella M, Pujol $R$, Callen $E$, et al. Origin, functional role, and clinical impact of Fanconi anemia FANCA mutations. Blood. 2011;117:3759-69.

13. Castella M, Pujol R, Callen E, et al. Chromosome fragility in patients with Fanconi anaemia: diagnostic implications and clinical impact. J Med Genet. 2011;48:242-50.

14. Mankad A, Taniguchi T, Cox B, et al. Natural gene therapy in monozygotic twins with Fanconi anemia. Blood. 2006;107:3084-90.

15. Asur RS, Kimble DC, Lach FP, et al. Somatic mosaicism of an intragenic FANCB duplication in both fibroblast and peripheral blood cells observed in a Fanconi anemia patient leads to milder phenotype. Mol Genet Genom Med. 2018;6:77-91.

16. Fanconi Anemia Research Fund I. Fanconi anemia: guidelines for diagnosis and management. 4th ed. 2014

17. Alter $B P$, Joenje $H$, Oostra $A B$, et al. Fanconi anemia: adult head and neck cancer and hematopoietic mosaicism. Arch Otolaryngol Head Neck Surg. 2005:131:635-9.

18. Jensen TK, Jacobsen R, Christensen K, et al. Good semen quality and life expectancy: a cohort study of 43,277 men. Am J Epidemiol. 2009;170: $559-65$.

19. Eisenberg ML, Li S, Brooks JD, et al. Increased risk of cancer in infertile men: analysis of U.S. claims data. J Urol. 2015;193:1596-601.

20. Latif T, Kold Jensen T, Mehlsen J, et al. Semen quality is a predictor of subsequent morbidity. A Danish cohort study of 4,712 men with longterm follow-up. Am J Epidemiol. 2017;186:910-917.

21. Maduro MR, Casella R, Kim E, et al. Microsatellite instability and defects in mismatch repair proteins: a new aetiology for Sertoli cell-only syndrome. Mol Hum Reprod. 2003;9:61-8. 